\title{
Load Flow Computations in Different Coordinate Systems for a Power System with UPFC
}

\author{
Tomasz Okon ${ }^{1}$, Kazimierz Wilkosz ${ }^{1}$ \\ ${ }^{I}$ Wroclaw University of Technology, \\ Wybrzeze Wyspianskiego 27, 50-370 Wroclaw, Poland \\ kazimierz.wilkosz@pwr.wroc.pl
}

\begin{abstract}
This paper deals with the Newton-Raphson load flow solution for a power system with embedded UPFC, when this device is modeled with the use of so-called Voltage Source Model. The aim of the paper is to present results of the original investigation of the mentioned load flow solution from the numerical point of view. The carried out investigation give the base for the statement that performing the load flow computations in the rectangular coordinate system is much more beneficial than in the polar coordinate system, which is most often used in the existing papers.
\end{abstract}

Index Terms-Flexible AC transmission systems; load flow; power systems.

\section{INTRODUCTION}

Load Flow Solution (LFS) methods belong to the most performed ones in analyses of a Power System (PS). The most important requirements imposed on them are reliability and computing time. Now, there are many LFS methods. However, one still observes changes in modern PSs. Also in such cases an efficient solution of a load flow problem should be possible.

Many interesting papers describe LFS for PS with Unified Power Flow Controller (UPFC). Seeking for the possibly best solution of the mentioned LF, different models of UPFC are utilized. In [1], a UPFC device is represented by an ideal Synchronous Voltage Source (SVS), which can inject a voltage with a variable magnitude and angle. This source is in series with a power line. The SVS model can be only used when the assumption about an infinite busbar, to which the UPFC shunt converter is connected, is valid. The UPFC model considered in [2] and [3] has no such limitations. That model consists of two sources, i.e. a real voltage source in the series branch and an ideal current source in the shunt branch. In [4]-[11], there are taken into account two voltage sources in the UPFC model. One of them is in the series branch, the second one is in the shunt branch. In each branch there is also an admittance. Such the model is often referred to as Voltage Source Model (VSM). This is a completely general model. When VSM is utilized in the Newton-

Manuscript received 7 June, 2015; accepted 25 February, 2016.
Raphson (N-R) load flow algorithm, the UPFC state variables are considered in the same way as the nodal power network state variables. In consequence, the interaction between the network and UPFC is properly modelled. In [12], it is underlined that VSM introduces some difficulties in modelling PS with embedded UPFCs. The reason is the presence of the voltage sources. In effect, an admittance matrix has larger size and symmetry properties of this matrix are lost. There are not such consequences, when the Power Injection Model (PIM) [9], [12]-[14] or Shunt Admittance Model (SAM) [12] is taken into consideration. In PIM, the power injections of the shunt and series converter of UPFC are interpreted as node injections. In SAM, UPFC is represented by $\Pi$-section with two shunt admittances. Convergence speed of the N-R algorithm using PIM or SAM is higher than it is in the case of using VSM [12]. The Current Based Model (CBM), assuming the current on the series branch of UPFC as a control variable, is proposed in [15]. CBM allows more easily taking into account a current limitation of UPFC. Results of Load Flow Computations (LFCs) with use of CBM are comparable with the case of using PIM [15].

Analysing existing LFSs for PS with UPFC, one can observe lack of investigation of features of such the solution from the view-point of the used coordinate system. In the paper, results of the indicated investigation for the case of using the general model of UPFC, i.e. VSM, are presented. The investigation deals with the numerical features of the $\mathrm{N}$ R LFS. Knowledge of the mentioned features enables better programming LFC and in an extreme case it allows to avoid lack of results of this computations.

At the beginning of the further part of the paper, a general description of LFCs based on the N-R method is given. Then, consequences of the assumed model of UPFC in LFCs are outlined. In the paper, it is noticed, that in some cases of LFCs, the Jacobian matrix (utilized in the calculations) can be singular or ill-conditioned. Analysis of the conditionality of the Jacobian matrix before an iteration process starts and during this process is carried out. Number of iterations in LFCs is also considered. LFCs in the Polar Coordinate System (PCS) and in Rectangular Coordinate System (RCS) are taken into account. 


\section{A GENERAL DESCRIPTION OF LFCS BASED ON THE NEWTON-RAPHSON METHOD}

The N-R method solves iteratively a set of nonlinear equations which can be written as

$$
\mathbf{F}(\mathbf{x})=\mathbf{0}
$$

where $\mathbf{F}$ represents the set of $n$ nonlinear equations, and $\mathbf{x}$ is a vector of $n$ unknown state variables. Linearization of this problem is formulated as

$$
\mathbf{F}(\mathbf{x})=-\mathbf{J} \times \Delta \mathbf{x}
$$

where $\Delta \mathbf{x}$ is a correction of the vector $\mathbf{x}$. The elements of the square Jacobian matrix $\mathbf{J}$ are defined as $J_{i k}(\mathbf{x})=\partial f_{i}(\mathbf{x}) / \partial x_{k}, i$, $k=1,2, \ldots, n$.

In PCS, $\mathbf{x}=\left[\delta_{2}, \delta_{3}, \ldots \delta_{n}, V_{1}, V_{2}, \ldots V_{n}\right]^{\mathrm{T}}$, where: $V_{i}, \delta_{i}$ are a magnitude and phase angle of $\bar{V}_{i}$ (the voltage at the bus $i$ ) $i \in\{1,2, \ldots, n\}$, respectively. The bus 1 is considered as a reference bus and $\delta_{1}=0$. In PCS, (2) can be formulated as (Case 1):

$$
\left[\begin{array}{c}
\Delta \mathbf{P} \\
\Delta \mathbf{Q}
\end{array}\right]=-\left[\begin{array}{cc}
\frac{\partial \mathbf{P}}{\partial \boldsymbol{\delta}} & \frac{\partial \mathbf{P}}{\partial \mathbf{V}} \\
\frac{\partial \mathbf{Q}}{\partial \boldsymbol{\delta}} & \frac{\partial \mathbf{Q}}{\partial \mathbf{V}}
\end{array}\right] \times\left[\begin{array}{c}
\Delta \boldsymbol{\delta} \\
\Delta \mathbf{V}
\end{array}\right],
$$

or, as it is presented in [6], [16] (Case 2):

$$
\left[\begin{array}{c}
\Delta \mathbf{P} \\
\Delta \mathbf{Q}
\end{array}\right]=-\left[\begin{array}{cc}
\frac{\partial \mathbf{P}}{\partial \boldsymbol{\delta}} & \frac{\partial \mathbf{P}}{\partial \mathbf{V}} \mathbf{V} \\
\frac{\partial \mathbf{Q}}{\partial \boldsymbol{\delta}} & \frac{\partial \mathbf{Q}}{\partial \mathbf{V}} \mathbf{V}
\end{array}\right] \times\left[\begin{array}{c}
\Delta \boldsymbol{\delta} \\
\frac{\Delta \mathbf{V}}{\mathbf{V}}
\end{array}\right],
$$

where $\mathbf{P}=\left[P_{1}, P_{2}, \ldots P_{n}\right]^{\mathrm{T}}, \mathbf{Q}=\left[Q_{1}, Q_{2}, \ldots Q_{n}\right]^{\mathrm{T}}, \boldsymbol{\delta}=\left[\delta_{2}, \delta_{3}, \ldots\right.$ $\left.\delta_{n}\right]^{\mathrm{T}}, \mathbf{V}=\left[\begin{array}{lll}V_{1}, & V_{2}, \ldots & V_{n}\end{array}\right]^{\mathrm{T}}, P_{i}, Q_{i}$ are an active and reactive power injection at the bus $i$, respectively.

Modification presented in (4) leads to useful simplifying in computations of derivatives.

In RCS, $\mathbf{x}=\left[e_{1}, e_{2}, \ldots e_{n}, f_{2}, f_{3} \ldots f_{n}\right]^{\mathrm{T}}$, where $e_{i}, f_{l}$ are real and imaginary parts of $\bar{V}_{i}$, respectively; $f_{1}=0$, and (2) can be formulated as:

$$
\left[\begin{array}{c}
\Delta \mathbf{P} \\
\Delta \mathbf{Q}
\end{array}\right]=-\left[\begin{array}{cc}
\frac{\partial \mathbf{P}}{\partial \mathbf{e}} & \frac{\partial \mathbf{P}}{\partial \mathbf{f}} \\
\frac{\partial \mathbf{Q}}{\partial \mathbf{e}} & \frac{\partial \mathbf{Q}}{\partial \mathbf{f}}
\end{array}\right] \times\left[\begin{array}{c}
\Delta \mathbf{e} \\
\Delta \mathbf{f}
\end{array}\right],
$$

where $\mathbf{e}=\left[e_{1}, e_{2}, \ldots e_{n}\right]^{\mathrm{T}}, \mathbf{f}=\left[f_{2}, f_{3}, \ldots f_{n}\right]^{\mathrm{T}}$.

For both the coordinate systems we have

$$
P_{i}-j Q_{i}=V_{i} \times \mathbf{Y}_{\text {row } i} \mathbf{V}
$$

where $\mathbf{Y}_{\text {row } i}$ - the row $i$ of an admittance matrix.

The essential feature of LFCs in PCS is existence transcendental functions in (1). For these functions, the Taylor series is an infinite one. In RCS, in the considered formulas, we have only quadratic terms. This fact leads to significant simplification of an expansion in Taylor series for $\mathbf{F}(\mathbf{x})$.

\section{A MODEL OF UPFC}

An equivalent circuit of UPFC is presented in Fig. 1 [1].

UPFC is able to provide simultaneous real-time control of the voltage phasor at a distinguished bus and the impedance of a branch, in which UPFC operates, determining the active and reactive power flowing through the mentioned branch. In the paper, we consider the control of voltage magnitude at the bus, to which UPFC is connected and also the active and reactive power on the branch with UPFC.

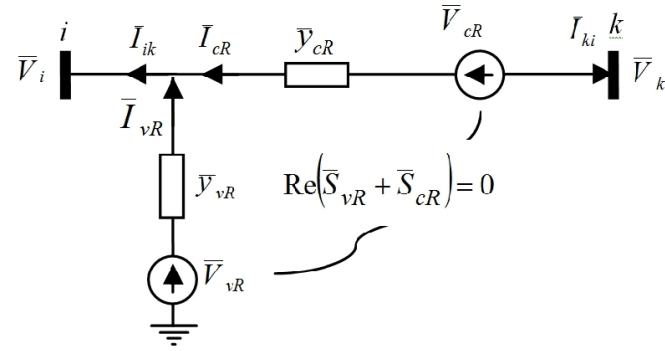

Fig. 1. Equivalent circuit of UPFC.

In the model, $V_{x}$ and $\delta_{x}$ are the controllable magnitude and angle, respectively, of the voltage $\bar{V}_{x}$, where $x \in\{c R, v R\}$.

Taking into account the equivalent circuit shown in Fig. 1 we can write:

$$
\begin{aligned}
& \bar{S}_{i k}^{*}=\left[-\left(\bar{y}_{v R}+\bar{y}_{c R}\right), \quad \bar{y}_{c R}, \quad \bar{y}_{c R}, \quad \bar{y}_{v R}\right] \times \\
& \times\left[V_{i}^{2}, \quad \bar{V}_{k} \times \bar{V}_{i}^{*}, \quad \bar{V}_{c R} \times \bar{V}_{i}^{*}, \quad \bar{V}_{v R} \times \bar{V}_{i}^{*}\right]^{T}, \\
& \bar{S}_{k i}^{*}=\left[\begin{array}{lll}
\bar{y}_{c R}, & -\bar{y}_{c R}, & -\bar{y}_{c R}
\end{array}\right] \times \\
& \times\left[\bar{V}_{i} \times \bar{V}_{k}^{*}, \quad V_{k}^{2}, \quad \bar{V}_{c R} \times \bar{V}_{k}^{*}\right]^{T}, \\
& \bar{S}_{c R}^{*}=\left[\begin{array}{lll}
-\bar{y}_{c R}, & \bar{y}_{c R}, & \bar{y}_{c R}
\end{array}\right] \times \\
& \times\left[\bar{V}_{i} \times \bar{V}_{c R}^{*}, \quad \bar{V}_{k} \times \bar{V}_{c R}^{*}, \quad V_{c R}^{2}\right]^{T}, \\
& \bar{S}_{v R}^{*}=\left[\bar{y}_{v R},-\bar{y}_{v R}\right] \times\left[V_{v R}^{2}, \bar{V}_{i} \bar{V}_{v R}^{*}\right]^{T},
\end{aligned}
$$

where $S$ stands for a complex power.

Neglecting UPFC losses, we can state that UPFC cannot absorb and injects real power, i.e. $P_{b b}=\operatorname{Re}\left(\bar{S}_{v R}+\bar{S}_{c R}\right)=0$.

The power injections at the buses $i$ and $k$, between which UPFC operates, are modified as follows: $\bar{S}_{i}=\bar{S}_{i}^{(\mathrm{ac})}-\bar{S}_{i k}^{(\mathrm{UPFC})}, \bar{S}_{k}=\bar{S}_{k}^{(\mathrm{ac})}-\bar{S}_{k i}^{(\mathrm{UPFC})}$, where: $\bar{S}_{i}^{(\mathrm{ac})}, \bar{S}_{k}^{(\mathrm{ac})}$ are calculated using $(6) ; \bar{S}_{i k}^{(\mathrm{UPFC})}, \bar{S}_{k i}^{(\mathrm{UPFC})}$ are calculated using (7) and (8), respectively.

\section{CONSIDERING UPFC IN LFCS}

\section{A. Modification of the Vectors $\boldsymbol{F}(\boldsymbol{x}), \Delta \boldsymbol{x}$ and the Matrix $\boldsymbol{J}$}

Considering UPFC in LFCs implies modification some of the existing elements of $\mathbf{F}(\mathbf{x}), \Delta \mathbf{x}$ and $\mathbf{J}$ as well as insertion of new elements into them. Fragments of $\mathbf{F}(\mathbf{x}), \Delta \mathbf{x}$, and $\mathbf{J}$, i.e. $\mathbf{F}_{F},(\Delta \mathbf{x})_{F}$, and $\mathbf{J}_{F}$, which in LFCs are associated with UPFC 
and contain elements modified or new (comparing with those elements considered in (3)-(5)), are as follows:

- in PCS-Case 1:

$$
\begin{aligned}
& \mathbf{F}_{F}^{\mathrm{T}}=\left[\begin{array}{lllllll}
\Delta P_{i} & \Delta P_{k} & \Delta Q_{i} & \Delta Q_{k} & \Delta P_{b b} & \Delta P_{k i} & \Delta Q_{k i}
\end{array}\right] \\
& (\Delta \mathbf{x})_{F}^{\mathrm{T}}=\left[\begin{array}{lllllll}
\Delta \delta_{i} & \Delta \delta_{k} & \Delta V_{k} & \Delta \delta_{v R} & \Delta \delta_{c R} & \Delta V_{v R} & \Delta V_{c R}
\end{array}\right],(12) \\
& \mathbf{J}_{F}=\left[\begin{array}{lllllll}
\frac{\partial P_{i}}{\partial \delta_{i}} & \frac{\partial P_{i}}{\partial \delta_{k}} & \frac{\partial P_{i}}{\partial V_{k}} & \frac{\partial P_{i}}{\partial \delta_{v R}} & \frac{\partial P_{i}}{\partial \delta_{c R}} & \frac{\partial P_{i}}{\partial V_{v R}} & \frac{\partial P_{i}}{\partial V_{c R}} \\
\frac{\partial P_{k}}{\partial \delta_{i}} & \frac{\partial P_{k}}{\partial \delta_{k}} & \frac{\partial P_{k}}{\partial V_{k}} & 0 & \frac{\partial P_{k}}{\partial \delta_{c R}} & 0 & \frac{\partial P_{k}}{\partial V_{c R}} \\
\frac{\partial Q_{i}}{\partial \delta_{i}} & \frac{\partial Q_{i}}{\partial \delta_{k}} & \frac{\partial Q_{i}}{\partial V_{k}} & \frac{\partial Q_{i}}{\partial \delta_{v R}} & \frac{\partial Q_{i}}{\partial \delta_{c R}} & \frac{\partial Q_{i}}{\partial V_{v R}} & \frac{\partial Q_{i}}{\partial V_{c R}} \\
\frac{\partial Q_{i}}{\partial P_{k b}} & \frac{\partial Q_{k}}{\partial V_{k}} & 0 & \frac{\partial Q_{k}}{\partial \delta_{c R}} & 0 & \frac{\partial Q_{k}}{\partial V_{c R}} \\
\frac{\partial \delta_{i}}{\partial \delta_{k}} & \frac{\partial P_{b b}}{\partial V_{k}} & \frac{\partial P_{b b}}{\partial \delta_{k}} & \frac{\partial P_{b b}}{\partial \delta_{c R}} & \frac{\partial P_{b b}}{\partial V_{v R}} & \frac{\partial P_{b b}}{\partial V_{c R}} \\
\frac{\partial P_{k i}}{\partial \delta_{i}} & \frac{\partial P_{k i}}{\partial \delta_{k}} & \frac{\partial P_{k i}}{\partial V_{k}} & 0 & \frac{\partial P_{k i}}{\partial \delta_{c R}} & 0 & \frac{\partial P_{k i}}{\partial V_{c R}} \\
\frac{\partial Q_{k i}}{\partial \delta_{i}} & \frac{\partial Q_{k i}}{\partial \delta_{k}} & \frac{\partial Q_{k i}}{\partial V_{k}} & 0 & \frac{\partial Q_{k i}}{\partial \delta_{c R}} & 0 & \frac{\partial Q_{k i}}{\partial V_{c R}}
\end{array}\right]
\end{aligned}
$$

- in PCS-Case 2:

$$
\begin{aligned}
& \mathbf{F}_{F}^{\mathrm{T}}=\left[\begin{array}{lllllll}
\Delta P_{i} & \Delta P_{k} & \Delta Q_{i} & \Delta Q_{k} & \Delta P_{b b} & \Delta P_{k i} & \Delta Q_{k i}
\end{array}\right] \\
& (\Delta \mathbf{x})_{F}^{\mathrm{T}}=\left[\begin{array}{lllllll}
\Delta \delta_{i} & \Delta \delta_{k} & \frac{\Delta V_{k}}{V_{k}} & \Delta \delta_{v R} & \Delta \delta_{c R} & \frac{\Delta V_{v R}}{V_{v R}} & \frac{\Delta V_{c R}}{V_{c R}}
\end{array}\right]
\end{aligned}
$$

In Case 2, only the elements of $\mathbf{J}_{F}$ in the columns numbered 3, 6, and 7 are different from the appropriate elements of $\mathbf{J}_{F}$ in Case 1. One can write: $X_{3}^{(2)}=X_{3}^{(1)} V_{k}$, $X_{6}^{(2)}=X_{6}^{(1)} V_{v R}$, and $X_{7}^{(2)}=X_{7}^{(1)} V_{c R}$, where the superscript of $X$ denotes the considered case and the subscript of $X$ is the number of the column in $\mathbf{J}_{F} ; X$ is any element of the indicated column of $\mathbf{J}_{F}$.

- in RCS:

$$
\begin{aligned}
& \mathbf{F}_{F}^{\mathrm{T}}=\left[\begin{array}{llllllll}
\Delta P_{i} & \Delta P_{k} & \Delta Q_{i} & \Delta Q_{k} & \Delta P_{b b} & \Delta P_{k i} & \Delta Q_{k i} & V_{i}^{2}
\end{array}\right], \\
& (\Delta \mathbf{x})_{F}^{\mathrm{T}}=\left[\begin{array}{llllllll}
\Delta e_{i} & \Delta e_{k} & \Delta f_{i} & \Delta f_{k} & \Delta e_{v R} & \Delta e_{c R} & \Delta f_{v R} & \Delta f_{c R}
\end{array}\right],(17) \\
& {\left[\begin{array}{llllllll}
\frac{\partial P_{i}}{\partial e_{i}} & \frac{\partial P_{i}}{\partial e_{k}} & \frac{\partial P_{i}}{\partial f_{i}} & \frac{\partial P_{i}}{\partial f_{k}} & \frac{\partial P_{i}}{\partial e_{v R}} & \frac{\partial P_{i}}{\partial e_{c R}} & \frac{\partial P_{i}}{\partial f_{v R}} & \frac{\partial P_{i}}{\partial f_{c R}}
\end{array}\right]} \\
& \frac{\partial P_{k}}{\partial e_{i}} \quad \frac{\partial P_{k}}{\partial e_{k}} \quad \frac{\partial P_{k}}{\partial f_{i}} \quad \frac{\partial P_{k}}{\partial f_{k}} \quad 0 \quad \frac{\partial P_{k}}{\partial e_{c R}} \quad 0 \quad \frac{\partial P_{k}}{\partial f_{c R}}
\end{aligned}
$$

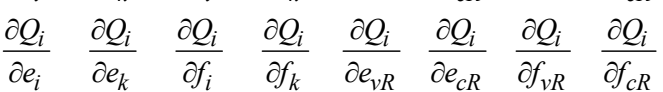

$$
\begin{aligned}
& \mathbf{J}_{F}=\left|\begin{array}{cccccccc}
\frac{\partial Q_{k}}{\partial e_{i}} & \frac{\partial Q_{k}}{\partial e_{k}} & \frac{\partial Q_{k}}{\partial f_{i}} & \frac{\partial Q_{k}}{\partial f_{k}} & 0 & \frac{\partial Q_{k}}{\partial e_{c R}} & 0 & \frac{\partial Q_{k}}{\partial f_{c R}} \\
\frac{\partial P_{b b}}{\partial e_{i}} & \frac{\partial P_{b b}}{\partial e_{k}} & \frac{\partial P_{b b}}{\partial f_{i}} & \frac{\partial P_{b b}}{\partial f_{k}} & \frac{\partial P_{b b}}{\partial e_{v R}} & \frac{\partial P_{b b}}{\partial e_{c R}} & \frac{\partial P_{b b}}{\partial f_{v R}} & \frac{\partial P_{b b}}{\partial f_{c R}}
\end{array}\right| . \\
& \frac{\partial P_{k i}}{\partial e_{i}} \quad \frac{\partial P_{k i}}{\partial e_{k}} \quad \frac{\partial P_{k i}}{\partial f_{i}} \quad \frac{\partial P_{k i}}{\partial f_{k}} \quad 0 \quad \frac{\partial P_{k i}}{\partial e_{c R}} \quad 0 \quad \frac{\partial P_{k i}}{\partial f_{c R}} \\
& \frac{\partial Q_{k i}}{\partial e_{i}} \frac{\partial Q_{k i}}{\partial e_{k}} \quad \frac{\partial Q_{k i}}{\partial f_{i}} \quad \frac{\partial Q_{k i}}{\partial f_{k}} \quad 0 \quad \frac{\partial Q_{k i}}{\partial e_{c R}} \quad 0 \quad \frac{\partial Q_{k i}}{\partial f_{c R}} \\
& {\left[\begin{array}{llllllll}
\frac{\partial V_{i}^{2}}{\partial e_{i}} & 0 & \frac{\partial V_{i}^{2}}{\partial f_{i}} & 0 & 0 & 0 & 0 & 0
\end{array}\right]}
\end{aligned}
$$

\section{B. Problematic Elements of the Jacobian Matrix}

The elements of the matrix $\mathbf{J}$ are determined by the bus voltages, the voltages $\bar{V}_{c R}, \bar{V}_{v R}$, and admittances charactering particular branches of the PS model and the model of UPFC. It can be noticed that magnitudes of every bus voltage and the voltage $\bar{V}_{v R}$, are approximately equal to 1 p.u. (usually between 0.9 and 1.1 p.u.). $V_{c R}$ may change from zero to 0.2 p.u. [17]. Small values of $V_{c R}$ may lead to small values of certain elements of $\mathbf{J}$ and in consequence to a significant increase of the condition number of this matrix. The elements of $\mathbf{J}$, which depend on $V_{c R}$ are calculated taking into account the following formulas:

- in PCS:

$$
\begin{aligned}
& \frac{\partial \bar{S}_{i k}^{*}}{\partial \delta_{c R}}=\overline{\mathrm{j}}_{c R} V_{c R} V_{i} \mathrm{e}^{\mathrm{j}\left(\delta_{c R}-\delta_{i}\right)}, \frac{\partial \bar{S}_{i k}^{*}}{\partial V_{c R}}=\bar{y}_{c R} V_{i} \mathrm{e}^{\mathrm{j}\left(\delta_{c R}-\delta_{i}\right)}, \\
& \frac{\partial \bar{S}_{k i}^{*}}{\partial \delta_{c R}}=-\mathrm{j} \bar{y}_{c R} V_{c R} V_{k} \mathrm{e}^{\mathrm{j}\left(\delta_{c R}-\delta_{k}\right)}, \frac{\partial \bar{S}_{k i}^{*}}{\partial V_{c R}}=-\bar{y}_{c R} V_{k} \mathrm{e}^{\mathrm{j}\left(\delta_{c R}-\delta_{k}\right)}, \\
& \frac{\partial \bar{S}_{b b}^{*}}{\partial \delta_{c R}}=\overline{\mathrm{j}}_{c R} V_{c R}\left(V_{i} \mathrm{e}^{\mathrm{j}\left(\delta_{i}-\delta_{c R}\right)}-V_{k} \mathrm{e}^{\mathrm{j}\left(\delta_{k}-\delta_{c R}\right)}\right), \\
& \frac{\partial \bar{S}_{b b}^{*}}{\partial V_{c R}}=-\bar{y}_{c R}\left(V_{i} \mathrm{e}^{\mathrm{j}\left(\delta_{i}-\delta_{c R}\right)}-V_{k} \mathrm{e}^{\mathrm{j}\left(\delta_{k}-\delta_{c R}\right)}-2 V_{c R}\right) .
\end{aligned}
$$

- in RCS:

$$
\begin{aligned}
& \frac{\partial \bar{S}_{i k}^{*}}{\partial e_{c R}}=\bar{y}_{c R} \bar{V}_{i}^{*}, \quad \frac{\partial \bar{S}_{i k}^{*}}{\partial f_{c R}}=\mathrm{j} \bar{y}_{c R} \bar{V}_{i}^{*}, \quad \frac{\partial \bar{S}_{k i}^{*}}{\partial e_{c R}}=-\bar{y}_{c R} \bar{V}_{k}^{*}, \\
& \frac{\partial \bar{S}_{k i}^{*}}{\partial f_{c R}}=-\mathrm{j} \bar{y}_{c R} \bar{V}_{k}^{*}, \quad \frac{\partial \bar{S}_{b b}^{*}}{\partial e_{c R}}=-\bar{y}_{c R}\left(\bar{V}_{i}-\bar{V}_{k}-2 e_{c R}\right), \\
& \frac{\partial \bar{S}_{b b}^{*}}{\partial f_{c R}}=\bar{y}_{c R}\left(\mathrm{j} \bar{V}_{i}-\mathrm{j} \bar{V}_{k}+2 f_{c R}\right) .
\end{aligned}
$$

It can be seen, that when LFCs are performed in PCS, and if $V_{c R}$ is equal to zero, all elements of $\mathbf{J}_{F}$, dependent on this voltage, are also equal to zero. Hence all elements in the fifth column of $\mathbf{J}_{F}$ in Case 1 and Case 2 are zero and $\mathbf{J}_{F}$ is not a full-rank matrix. In Case 2, the situation is even worse because additionally elements in the seventh column of $\mathbf{J}_{F}$ are equal to zero, as well. This problem does not exist if $\mathrm{RCS}$ is taken into consideration.

\section{INITIAL CONDITIONS FOR LFCS}

Proper starting conditions are important in any iterative process. For the simple case, in which no UPFC is present, for all $P Q$ buses the suitable starting point is 1 for voltage magnitudes and 0 for voltage angles. However, if a UPFC device is considered, the following initial conditions are proposed [6]:

$$
\begin{gathered}
\delta_{c R}^{0}=\arctan \left(P_{k i r e f} / c\right), \\
V_{c R}^{0}=x_{c R} \sqrt{P_{k i r e f}^{2}+c^{2}} / V_{k}^{0}, \\
\delta_{v R}^{0}=-\arcsin \left(\frac{\left(V_{i}^{0}-V_{k}^{0}\right) V_{c R}^{0} x_{v R} \sin \delta_{c R}^{0}}{V_{v R}^{0} V_{i}^{0} x_{c R}}\right),
\end{gathered}
$$


where $c=Q_{k i r e f}-V_{k}^{0}\left(V_{k}^{0}-V_{i}^{0}\right) / x_{c R} ; P_{k i r e f}$ is a reference power flow on the branch between the nodes $i$ and $k$ at the node $k ; x_{c R}, x_{v R}$ are the inductive reactances, respectively, in the series and in the shunt branch in the UPFC model (Fig. 1).

If the UPFC shunt converter keeps $V_{i}$ on a fixed value, $V_{v R}$ is initialized by the target voltage value at the bus $i$.

\section{THE CONDITION NUMBER OF THE JACOBIAN MATRIX IN LFCS}

\section{A. Definition of the Condition Number}

LFCs based on the N-R method lead to iterative solving the linear problem which can be formulated as seeking a solution of an equation $\mathbf{A u}=\mathbf{b}$, where $\mathbf{u}$ is a vector of unknowns, $\mathbf{A}$ is a matrix of coefficients, $\mathbf{b}$ is a vector of known values. In the considered LFCs, $\mathbf{u}=\Delta \mathbf{x}, \mathbf{A}=-\mathbf{J}$, $\mathbf{b}=\mathbf{F}(\mathbf{x})$. For certain values of elements of the matrix $\mathbf{A}$ and the vector $\mathbf{b}$, one obtains the vector $\mathbf{u}$. When there are slight changes of elements of $\mathbf{b}$ we obtain the vector $\mathbf{u}$ '. The question is "How much the calculated vector is different?", i.e. what is the sensitivity of the solution of the considered problem to (slight) changes of the elements of b. The mentioned sensitivity has essential influence on $\Delta \mathbf{x}$ and also on convergence of the iterative process of LFCs. The strong sensitivity of $\Delta \mathbf{x}$ to changes of the elements of $\mathbf{F}(\mathbf{x})$ implies increasing a number of iterations of computations and thereby increasing a cost of solving a load flow problem.

The stronger the sensitivity of $\Delta \mathbf{x}$ to variations of the elements of $\mathbf{F}(\mathbf{x})$ is, the larger the so-called condition number $\kappa(\mathbf{J})$ is. The condition number $\kappa(\mathbf{J})$ can be calculated as $\kappa(\mathbf{J})=\|\mathbf{J}\| \mathbf{J}^{-1} \|[17]$, where: \|\| denotes a matrix norm. In the paper, we use the spectral norm of $\mathbf{J}$.

\section{B. The Condition Number of the Jacobian Matrix for Different Coordinate Systems}

The analysis in the section IV shows that the voltage $\bar{V}_{c R}$ has different influence on elements of the Jacobian matrix $\mathbf{J}$ in different coordinate systems. In this section, the results of quantitative investigations of that influence are presented. The aim of the investigations is determination of quantitative dependence of the condition number $\kappa(\mathbf{J})$ on: (i) the initial value of the magnitude of the voltage $\bar{V}_{c R}$ in LFCs $\left(V_{c R, 0}\right)$, (ii) the actual magnitude of the voltage $\bar{V}_{c R}$, i.e. the magnitude of the voltage $\bar{V}_{c R}$, being the result of LFCs $\left(V_{c R}\right)$. During the performed investigations the following assumptions are made:

1. The IEEE-14 bus test PS is considered [18].

2. UPFC is installed on the line between the buses 4 and 5. The shunt branch of UPFC is connected with the bus 5 .

3. Power injections and loads in the test PS are not changed (regarding to the base case).

4. The shunt inverter of UPFC operates as a voltage regulator (the magnitude of the voltage $\bar{V}_{5}$, i.e. the voltage at the node 5 is constant).

5. Different values of $V_{c R, 0}$ and $V_{c R}$ are considered. The sets of values of $V_{c R, 0}$ and $V_{c R}$ are as follows: (i) for $V_{c R, 0}$ :
$\{0.001,0.01,0.1,0.2\}$ p.u. (ii) for $V_{c R}:\{0,0.001,0.01$, $0.1,0.2\}$ p.u.

6. During LFCs, the stopping criteria is $\max _{i}\left|f_{i}(\mathbf{x})\right|<10^{-12}$, or the number of iterations is equal to 50 .

Results of carried out LFCs are depicted in Fig. 2-Fig. 7.

In Fig. 2-Fig. 4, the condition number $\kappa(\mathbf{J})$ versus $V_{c R, 0}$ for PCS and RCS is presented. The investigations show that for both cases, which are distinguished, when PCS is used, results of computations of $\kappa(\mathbf{J})$ are approximately the same (the difference is not larger than $1.6 \%$ ). For PCS, $\kappa(\mathbf{J})$ is relatively large, when the magnitude $V_{c R, 0}$ adopts small values. When $V_{c R, 0} \leq 0.03$ p.u., $\kappa(\mathbf{J})>10^{3}$. For decreasing values of $V_{c R, 0}, \kappa(\mathbf{J})$ fast increases. For larger values of $V_{c R, 0}$, $\kappa(\mathbf{J})$ is smaller. For $V_{c R, 0}=0.2, \kappa(\mathbf{J})$ is close to 175 .

In the case of RCS, when $V_{c R, 0} \leq 0.03$ p.u., $\kappa(\mathbf{J}) \leq 181.16$. We can observe slowly increasing of $\kappa(\mathbf{J})$ with the increase of $V_{c R, 0}$. For the change of $V_{c R, 0}$ from 0.001 to 0.2 p.u., $\kappa(\mathbf{J})$ changes not more than $1 \%$.

For $V_{c R, 0} \approx 0.18, \kappa(\mathbf{J})$ for RCS becomes larger than $\kappa(\mathbf{J})$ for PCS. When $V_{c R, 0}>0.18$, the difference between values of $\kappa(\mathbf{J})$ for both considered coordinate systems is the largest for $V_{c R, 0}=0.2$ p.u. and is approximately equal to $3.5 \%$.

$\kappa(\mathbf{J})$ changes during LFCs. It depends on $V_{c R, 0}$ and also $V_{c R}$. Exemplary plots, presenting $\kappa(\mathbf{J})$ as functions of the number of iterations for different $V_{c R, 0}$ and $V_{c R}$, are depicted in Fig. 5-Fig. 7.

Figure 5 and Fig. 6 are related to LFCs in PCS. Figure 7 presents results, when RCS is considered. In this last case, influence of $V_{c R, 0}$ on $\kappa(\mathbf{J})$ can be practically neglected. Differences between the values of $\kappa(\mathbf{J})$ for different values of $V_{c R, 0}$ are not larger than $1 \%$. Considering LFCs in PCS, influence of values of $V_{c R, 0}$ on $\kappa(\mathbf{J})$ cannot be neglected. Especially, that influence is large when the difference between $V_{c R}$ and $V_{c R, 0}$ is large (Fig. 5 and Fig. 6).

For PCS, depending on values of $V_{c R, 0}$, values of $\kappa(\mathbf{J})$ for successive iterations in LFCs are larger or smaller from the value of $\kappa(\mathbf{J})$ calculated before the first iteration. For certain iteration, the value of $\kappa(\mathbf{J})$ is practically independent on values of $V_{c R, 0}$. That value of $\kappa(\mathbf{J})$ depends on the actual value of $V_{c R}$. During LFCs in RCS, $\kappa(\mathbf{J})$ become larger than before the first iteration. For the largest considered values of $V_{c R}$, the increase of $\kappa(\mathbf{J})$ is about $10 \%$.

The values of $\kappa(\mathbf{J})$, determined at the end of the calculation process, are larger for PCS than for RCS. For $V_{c R}=0.2$ p.u., the difference of the mentioned values is about $14 \%$. For $V_{c R}=0$ p.u., that difference is incomparably larger.

Summing up the presented results of the investigations, we can state, that: 1 . For PCS, $\kappa(\mathbf{J})$, calculated before the first iteration, strongly depends on $V_{c R, 0}$, if this voltage is sufficiently small. Such strong dependence is not observed for larger values of $V_{c R, 0}$ (Fig. 2). 2. From the view-point of $\kappa(\mathbf{J})$, calculated before the first iteration, if $V_{c R, 0}$ is sufficiently large, features of LFCs in PCS and in RCS are comparable (Fig. 2-Fig. 4). One cannot draw such conclusion if values of $\kappa(\mathbf{J})$, determined at the end of LFCs, 
are considered (Fig. 5-Fig. 7). 3. Analysing $\kappa(\mathbf{J})$, determined before the first iteration and also during LFCs, one can ascertain that LFCs in RCS are more advantageous than in PCS (Fig. 5-Fig. 7).

\section{Consequences of Deterioration of Conditionality of the Jacobian Matrix}

During the investigations, attention is paid also to numbers of iterations in different cases of LFCs. Those numbers of iterations in LFCs for fixed values of $V_{c R}$, when the phase angle of $\bar{V}_{c R}\left(\delta_{c R}\right)$ changes, are determined. The set of values of $\delta_{c R}$ is as follows: $j \times 15$ degrees $j=0,1,2, \ldots$, 23. Impact of the assumed values of $V_{c R, 0}$ on the mentioned numbers of the iterations is also taken into account. The values of $V_{c R, 0}$ and $V_{c R}$, which are considered in the investigations, are such as it is shown in subsection $B$.

Results of the investigations of the earlier-mentioned numbers of the iterations are collected in Table I and Table II. In those tables, there are parameters characterizing numbers of iterations in LFCs performed in PCS and RCS.

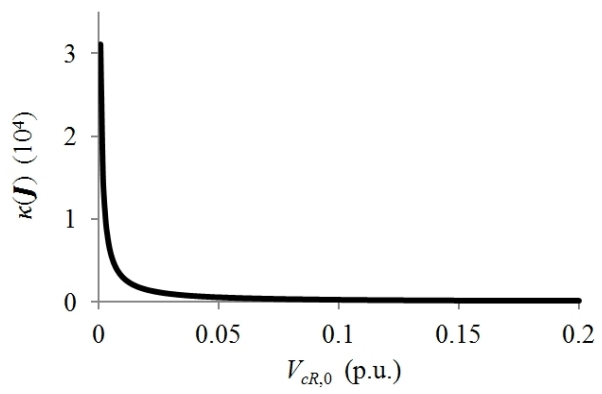

Fig. 2. The condition number of the matrix $\mathbf{J}$ versus $V_{c R, 0}$ in LFCs for PCS.

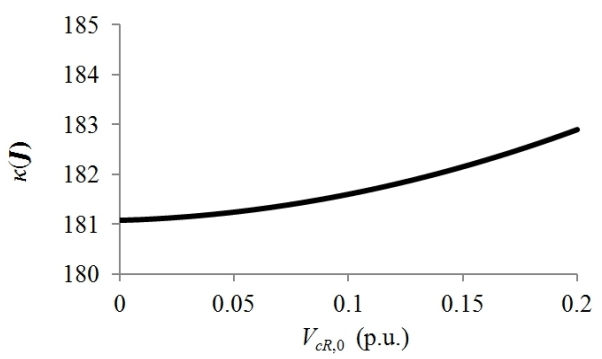

Fig. 3. The condition number of the matrix $\mathbf{J}$ versus $V_{c R, 0}$ in LFCs for RCS.

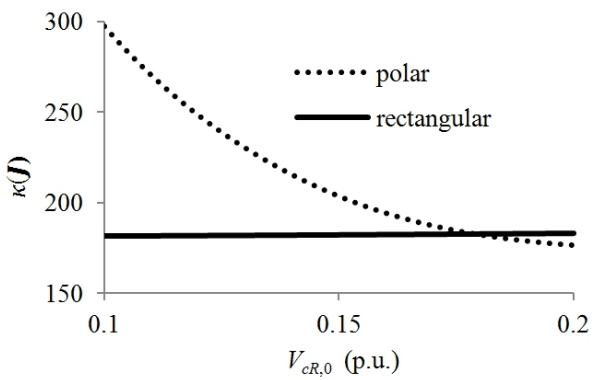

Fig. 4. The condition number of the matrix $\mathbf{J}$ versus $V_{c R, 0}$ in LFCs for different coordinate systems.

To characterize numbers of the iterations in LFCs the following parameters are used: (i) the minimum value $\left(m_{i t}\right)$, (ii) the maximum value $\left(M_{i t}\right)$, (iii) the arithmetic mean $\left(a_{i t}\right)$, (iv) the coefficient of variation $\left(C V_{R M S D, i t}=R M S D / a\right.$, where $R M S D$-the root-mean-square deviation).

Analysing Table I and Table II, we can ascertain that $m_{i t}=6$ for PCS as well as for RCS. Only in the case of PCS, when $V_{c R, 0}=0.001$ p.u and $V_{c R} \in\{0.1,0.2\}$ p.u $m_{i t}=7$. Other situation is, when the maximum number of iterations is taken into account. For RCS, one can distinguish such cases in which $M_{i t}=m_{i t}$. In other cases, at most $M_{i t}=8$. There is no case for PCS, in which $M_{i t}=m_{i t}$. In the most favorable case (for $V_{c R, 0}=0.01 \mathrm{p} . \mathrm{u}$ and $V_{c R}=0.01 \mathrm{p} . \mathrm{u}$ ), $M_{i t}=12$. One can find such cases, in which $M_{i t}=26$. In those cases, $M_{i t}-m_{i t}=20$. The mentioned value is a maximum value of $M_{i t}-m_{i t}$.

The coefficient $C V_{R M S D, \text { it }}$ for RCS is not larger than $10 \%$. For PCS, that coefficient is never less than $18 \%$. It achieves value even above $40 \%$. In many cases, defined by the values of $V_{c R}$ and $V_{c R, 0}, C V_{R M S D, i t}>30 \%$.

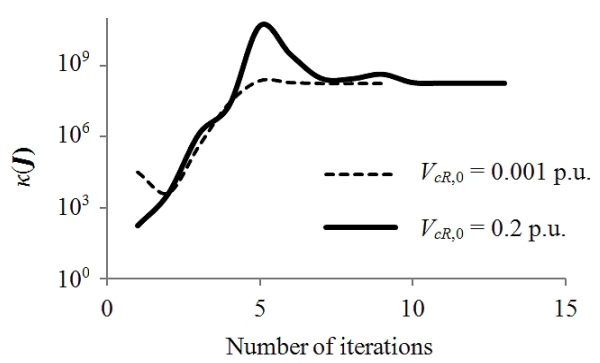

Fig. 5. The condition number of the matrix $\mathbf{J}$ versus the number of iterations for PCS and for different values of $V_{c R, 0}$, when $V_{c R}=0$ p.u.

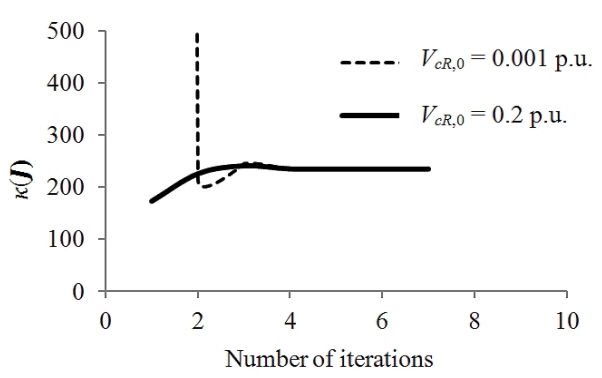

Fig. 6. The condition number of the matrix $\mathbf{J}$ versus the number of iterations for PCS and for different values of $V_{c R, 0}$, when $V_{c R}=0.2$ p.u.

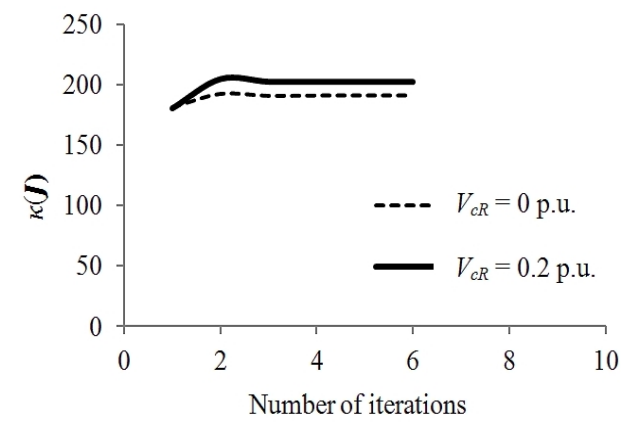

Fig. 7. The condition number of the matrix $\mathbf{J}$ versus the number of iterations for RCS, and for different values of the voltage magnitude $V_{c R}$.

Taking into account, the applied characteristics of numbers of the iterations in LFCs performed in PCS, one can notice, that there is no essential difference between the case of these computations, in which the value of $V_{c R, 0}$ is calculated using the formulas (20), and other ones.

In general, the analysis of numbers of the iterations in LFCs shows, that performing these computations in PCS is less beneficial than in RCS. For PCS, the arithmetic mean of numbers of iterations in LFCs is greater and variability of these numbers is much larger. This is a consequence of worse conditionality of the Jacobian matrix in PCS. 
TABLE I. CHARACTERISTICS OF NUMBERS OF ITERATIONS IN LFCS PERFORMED IN PCS.

\begin{tabular}{|c|c|c|c|c|c|}
\hline \multirow{2}{*}{ Parameter } & \multicolumn{5}{|c|}{$V_{c R}$, p.u. } \\
\hline & 0.001 & 0.01 & 0.1 & 0.2 & all \\
\hline \multicolumn{6}{|c|}{$V_{c R, 0}=0.001 \mathrm{p} . \mathrm{u}$} \\
\hline$m_{i t}$ & 6 & 6 & 7 & 7 & 6 \\
\hline$M_{i t}$ & 16 & 26 & 23 & 21 & 26 \\
\hline$a_{i t}$ & 10.25 & 10.33 & 9.54 & 9.83 & 9.98 \\
\hline$C V_{R M S D, i t}, \%$ & 27.63 & 43.34 & 35.91 & 30.97 & 34.53 \\
\hline \multicolumn{6}{|c|}{$V_{c R, 0}=0.01 \mathrm{p} . \mathrm{u}$} \\
\hline$m_{i t}$ & 6 & 6 & 6 & 6 & 6 \\
\hline$M_{i t}$ & 19 & 12 & 15 & 20 & 20 \\
\hline$a_{i t}$ & 9.50 & 8.46 & 9.38 & 10.00 & 9.34 \\
\hline$C V_{R M S D, i t}, \%$ & 35.12 & 20.62 & 27.39 & 31.76 & 29.72 \\
\hline \multicolumn{6}{|c|}{$V_{c R, 0}=0.1 \mathrm{p} . \mathrm{u}$} \\
\hline$m_{i t}$ & 7 & 6 & 6 & 6 & 6 \\
\hline$M_{i t}$ & 17 & 15 & 15 & 13 & 17 \\
\hline$a_{i t}$ & 9.17 & 8.50 & 8.21 & 8.54 & 8.64 \\
\hline$C V_{R M S D, i t}, \%$ & 26.66 & 22.48 & 25.65 & 18.26 & 23.70 \\
\hline \multicolumn{6}{|c|}{$V_{c R, 0}=0.2 \mathrm{p.u}$} \\
\hline$m_{i t}$ & 6 & 6 & 6 & 6 & 6 \\
\hline$M_{i t}$ & 16 & 18 & 16 & 13 & 18 \\
\hline$a_{i t}$ & 9.38 & 9.33 & 8.33 & 8.50 & 8.93 \\
\hline$C V_{R M S D, i t}, \%$ & 30.47 & 33.24 & 34.06 & 21.10 & 30.39 \\
\hline \multicolumn{6}{|c|}{$V_{c R, 0}-$ calculated } \\
\hline$m_{i t}$ & 6 & 6 & 6 & 6 & 6 \\
\hline$M_{i t}$ & 25 & 14 & 16 & 14 & 25 \\
\hline$a_{i t}$ & 9.29 & 7.88 & 9.25 & 9.17 & 8.89 \\
\hline$C V_{R M S D, i t}, \%$ & 40.45 & 25.76 & 31.92 & 24.43 & 31.86 \\
\hline
\end{tabular}

TABLE II. CHARACTERISTICS OF NUMBERS OF ITERATIONS IN LFCS PERFORMED IN RCS.

\begin{tabular}{|c|c|c|c|c|c|}
\hline \multirow{2}{*}{ Parameter } & \multicolumn{5}{|c|}{$V_{\boldsymbol{c R}, \text { p.u. }}$} \\
\cline { 2 - 6 } & 0.001 & 0.01 & 0.1 & 0.2 & all \\
\hline$m_{i t}$ & 6 & 6 & 6 & 6 & 6 \\
\hline$M_{i t}$ & 6 & 6 & 7 & 8 & 8 \\
\hline$a_{i t}$ & 6 & 6 & 6.25 & 6.46 & 6.18 \\
\hline$C V_{R M S D, i t} \%$ & 0 & 0 & 7.08 & 9.11 & 6.62 \\
\hline
\end{tabular}

\section{CONCLUSIONS}

Presence of UPFC in PS entails increasing a set of variables in a PS model. Among additional variables related with UPFC, a voltage of the source representing the series inverter $\left(\bar{V}_{c R}\right.$ in VSM), requires special attention.

The paper has contribution in LFCs. In the paper, it is noted that if the voltage magnitude $V_{c R}$ is equal to zero, then the Jacobian matrix (the matrix $\mathbf{J}$ ), which is utilized in LFCs performed in PCS, becomes singular. One should also expect deterioration of conditionality of the matrix $\mathbf{J}$ for values of $V_{c R}$, which are close to zero. It is shown, that other situation is when the mentioned computations are performed in RCS. In the paper, it is shown that conditionality of the matrix $\mathbf{J}$ before the first iteration in LFCs is slightly better for PCS than for RCS only for suitably large values of assumed initial voltage magnitude $V_{c R}$ (i.e. $V_{c R, 0}$ ). During computations, the conditionality of the matrix $\mathbf{J}$ changes and in no analysed case, in the final phase of computations, the considered conditionality is better for PCS than for RCS. In consequence, when that last coordinate system is used, the arithmetic mean of number of the iterations in LFCs is smaller and range of variability of this number is essentially less. The carried out investigations, utilizing the quantitative measures (the condition number of the matrix $\mathbf{J}$, the number of the iterations in LFCs) for evaluation of LFCs performed in different coordinate systems, allows to state that performing this calculation in RCS is significantly better than in PCS.

\section{REFERENCES}

[1] C. Fuerte-Esquivel, E. Acha, "A Newton-type algorithm for the control of power flow in electrical power networks", IEEE Trans. on Power Systems, vol. 12, no. 4, pp. 1474-1480, 1997. [Online] Available: http://dx.doi.org/10.1109/59.627844

[2] S.-H. Lee, C.-C. Chu, D.-H. Chang, "Comprehensive UPFC models for power flow calculations in practical power systems", in Proc. IEEE PES Summer Meeting, Vancouver, BC, 2001, vol. 1, pp. 2732.

[3] A. Karthikeyan, C. Nagamani, S. Srividhya, "Analysis of boundaries of controllable power flow with UPFC considering line loss", in Proc. The 3rd IEEE Conf. on Industrial Electronics and Appls, Singapore, 2008, pp. 2078-2083. [Online]. Available: http://dx.doi.org/10.1109/ iciea.2008.4582886

[4] A. Nabavi-Niaki, M. R. Iravani, "Steady-state and dynamic models of unified power flow controller (UPFC) for power system studies", IEEE Trans. on Power Syst., vol. 11, no. 4, pp. 1937-1943, 1996. [Online]. Available: http://dx.doi.org/10.1109/59.544667

[5] C. R. Fuerte-Esquivel, E. Acha, "Unified power flow controller: a critical comparison of Newton-Raphson UPFC algorithms in power flow studies", IEE Proc.-GTD, vol. 144, no. 5, 1997. [Online]. Available: http://dx.doi.org/10.1049/ip-gtd:19971385

[6] C. R. Fuerte-Esquivel, E. Acha, H. Ambriz-Perez, "A comprehensive Newton-Raphson UPFC model for the quadratic power flow solution of practical power networks", IEEE Trans. Power Syst., vol. 15, no. 1, pp. 102-109, 2000. [Online]. Available: http://dx.doi.org/ $10.1109 / 59.852107$

[7] A. M. M. Abdel-Rahim, N. P. Padhy, "Newton-Raphson UPFC model for power flow solution of practical power networks with sparse techniques", in Proc. Inter. Conf. on Electric Utility Deregulation, Restructuring and Power Technologies, Hong Kong, 2004, vol. 1, pp. 77-83. [Online]. Available: http://dx.doi.org/10.1109/DRPT.2004.1338472

[8] N. M. R. Santos, V. M. F. Pires, M. G. Castro, "A new model to incorporate unified power flow controllers in power flow studies", in Proc. IEEE PES Transmission and Distribution Conf. and Exhibition, Dallas, TX, 2006, pp. 133-140. [Online]. Available: http://dx.doi.org/10.1109/TDC.2006.1668472

[9] M. Z. El-Sadek, A. Ahmed, H. E. Zidan, "Comparison of unified power flow controller models for power flow studies", in Proc. Eleventh Int. Middle East Power Systems Conf. (MEPCON 2006), El-Minia, 2006, vol. 1, pp. 189-196.

[10] S. Bhowmick, B. Das, N. Kumar, "An indirect UPFC model to enhance reusability of newton power-flow codes", IEEE Trans. Power Delivery, vol. 23, no. 4, pp. 2079-2088, 2008. [Online]. Available: http://dx.doi.org/10.1109/TPWRD.2008.923105

[11] S. S. Shrawane, M. Diagavane, N. Bawane, "Optimal reactive power dispatch by furnishing UPFC using multi-objective hybrid GAPSO approach for transmission loss minimisation and voltage stability", in Proc. Inter. Conf. on Nascent Technologies in the Engineering Field, Navi Mumbai, 2015, pp. 1-6. [Online]. Available: http://dx.doi.org/10.1109/icnte.2015.7029923

[12] A. L'Abbate, M. Trovato, C. Becker, E. Handschin, "Advanced steady-state models of UPFC for power systems studies", in Proc. IEEE PES Summer Meeting, Chicago, IL, 2002, vol. 1, pp. 449-454. [Online]. Available: http://dx.doi.org/10.1109/PESS.2002.1043275

[13] E. Handschin, C. Lehmkoster, "Optimal power flow for deregulated systems with FACTS-devices", in Proc. Power Systems Computation Conference (PSCC), Trondheim, Norway, 1999, pp. 1270-1276.

[14] T. Qiang, J. Qiang, "A novel method of power flow analysis with UPFC considering limit violations of variable", in Proc. Inter. Conf. on Electric Information and Control Engg, Wuhan, 2011, pp. 70-74. [Online]. Available: http://dx.doi.org/10.1109/iceice.2011.5777609

[15] M. Pereira, Z. L. Cera, "A current based model for load flow studies with UPFC", IEEE Trans. on Power Syst., vol. 28, no. 2, pp. $677-$ 682, 2013. [Online]. Available: http://dx.doi.org/10.1109/TPWRS. 2012.2206409

[16] A. K. Sahoo, S. S. Dash, T. Thyagarajan, "Modeling of STATCOM and UPFC for power system steady state operation and control", in Proc. Information and Communication Technology in Electrical Sciences (ICTES), Chennai, Tamil Nadu, India, 2007, pp. 458-463. [Online]. Available: http://dx.doi.org/10.1049/ic:20070656

[17] E. Acha, FACTS: Modelling and simulation in power networks. Chichester: Wiley, 2004. [Online]. Available: http://dx.doi.org/ $10.1002 / 0470020164$

[18] Power Systems Test Case Archive. [Online]. Available: http://www.ee.washington.edu/research/pstca/ 